\title{
From Micro to Macro: Regulation Up- and Down-Adult Neurogenesis Process
}

\author{
A. C. Nunciato ${ }^{1 \#}$, E. L. Meirelles², Patricia Gardino² \\ ${ }^{1}$ Laboratório de Neuroanatomia Celular, \\ Instituto de Ciências Biomédicas, Universidade Federal do Rio de Janeiro, Rio de Janeiro, Brazil \\ ${ }^{2}$ Laboratório de Neurobiologia da Retina e Neuroquímica, \\ Instituto de Biofísica Carlos Chagas Filho, Universidade Federal do Rio de Janeiro, Rio de Janeiro, Brazil \\ Email: \#ananunciato@gmail.com
}

Received July 24, 2013; revised August 29, 2013; accepted September 15, 2013

Copyright (C) 2013 A. C. Nunciato et al. This is an open access article distributed under the Creative Commons Attribution License, which permits unrestricted use, distribution, and reproduction in any medium, provided the original work is properly cited.

\begin{abstract}
Neurogenesis in the adult brain has been a field of growing interest. There are two neurogenic niches in the mammalian adult brain: subgranular zone (SGZ) of the hippocampus and the subventricular zone (SVZ), producing new neurons that use the rostral migratory stream (RMS) to reach olfactory bulb (OB). Cells in these two discrete regions retain the capacity to generate multiple lineages in vitro and in vivo. Thus far, the mechanisms involved in the regulation of these cells have not been well elucidated, once the underlying cellular signaling mechanism is not fully understood. The aim of this work is to collect the most recent papers published in this field to elucidate the signaling pathways involved in the adult "up" and "down" neurogenesis. The most cited pathways included directly or indirectly associations with neurotrophins and transcription factors. This work provides a schema showing the outline from "macro" to "micro" signaling for neurogenesis in the SGZ of the hippocampus. It also includes a session linking neurogenesis to the pathophysiology of mood disorders and stress-related cognitive dysfunctions. Further characterization of these molecular components could provide greater insight into the mechanisms involved in the regulation of neurogenesis in the adult brain.
\end{abstract}

Keywords: Adult Neurogenesis; Hippocampus; SVZ; Signaling; Pathway

\section{Introduction}

Neurogenesis, a process of generating functionally integrated neurons from progenitor cells, was traditionally believed to occur only during embryonic stages in the mammalian central nervous system (CNS). Nowadays, it is well demonstrated that new neurons can be integrated in the mature CNS throughout life. Adult neurogenesis in the mammalian brain is generally considered as an active process encompassing the proliferation and cell fate specification of adult neural progenitors, and their subsequent differentiation, maturation, migration and functional integration into the existing neuronal circuitry $[1,2]$.

\section{Traditional Neurogenic Niches}

In the intact adult mammalian CNS, active neurogenesis occurs in two discrete "neurogenic" regions: the subgranular zone (SGZ) of the dentate gyrus (DG) in the

\footnotetext{
*All authors contributed equally for this work.

${ }^{\#}$ Corresponding author.
}

hippocampus (see Figure 1) and the subventricular zone (SVZ) of the lateral ventricles in the forebrain. Evidence has shown that the hippocampus is able to generate new neurons (i.e. neurogenesis) throughout the lifespan of mammals, including humans, changing our opinion about mechanisms underlying effects of current drugs $[1,3]$. The SGZ of the DG of the hippocampus is one of the two major sites of neurogenesis in the adult brain, along with the SVZ that continuously feeds new bulbar interneurons to the olfactory lobe via the rostral migratory stream [2].

The granule cell layer of the dentate gyrus of the SGZ in the in the adult brain contains neural progenitor cells (NPCs) capable of generating new neurons daily. These newborn neurons integrate into the existing circuitry. Moreover, the time-course of maturation of newly generated neurons in the DG is generally consistent with the delayed onset of therapeutic effects of current pharmacological treatment. The most compelling evidence linking adult hippocampal neurogenesis with antidepressants comes from recent studies that demonstrate suppression 
of hippocampal neurogenesis in the depressive disorder $[4,5]$.

The demonstration of active adult neurogenesis also opens possibilities to repair the adult CNS after injury or degenerative neurological diseases using cell replacement therapy in the near future. Furthermore, the hippocampus, as an integral component of the limbic system, is a focus of depression research and appears to serve as a primary site of action for antidepressants that inhibit pathological hyperactivity [6]. However, there is evidence suggesting that antidepressants can stimulate hippocampal activity and induce hippocampal neurogenesis noted through behavioral responses. Moreover, excitatory hippocampal neurons are injured by chronic stress and animal models have proven useful in identifying molecular and cellular markers relevant to depression [6].

Adult mammalian SVZ NPCs retain the capacity to generate multiple lineages in vitro and in vivo [7]. Thus far, the mechanisms involved in the regulation of these cells have not been well elucidated, once the underlying signaling mechanism is not fully understood.

Adult neurogenesis in the DG of the hippocampus has gained considerable attention as a cellular substrate for both the pathophysiology and treatment of depression [5].

Furthermore, in the adult brain, loss of neurogenic potential can lead to cognitive deficits and in combination with neural death can even contribute to progressive neurodegenerative disease [8]. Adult hippocampal neurogenesis plays an important role in learning and memory processes and its abnormal regulation might account for cognitive impairments associated with Alzheimer Disease and other dementia [9].

The aim of this study is to contribute to elucidate the underling biomolecular mechanisms of up- and downneurogenesis processes, identifying pathways that regulate progenitor cell proliferation and to define a set of transcripts that can be used as molecular tools in the drug discovery process [7]. The significance of these discoveries is presented in the context of human brain disorders and how this knowledge could contribute to pharmacotherapeutic interventions targeting signaling aimed at treating such diseases. Unraveling these precise molecular genetic networks is crucial to understanding how neural stem and progenitor cells function [8]. We processed a review in the recent literature, using neurogenesis, hippocampus, subventricular zone (SVZ), signaling, pathway, adult neurogenesis, as searching words in PUBMED database. Only papers published from 2006 to date were included. The first explore returned two hundred sixty seven papers. The abstracts were further analyzed and 26 articles were finally selected to this minireview.

\section{Micro: Signaling Pathway: Up- and Down-Neurogenesis}

The adult SVZ contains neural stem cells (NSC) that generate neuroblasts migrating to the olfactory bulb (OB) and differentiating into interneurons. The molecular cues controlling essential functions within the neurogenesis pathway such as proliferation, short and long distance migration, functional integration and cell survival are poorly understood [10].

It is well established that there are pathways that regulate up- and down-neurogenesis. Below we quote the molecular mechanisms associated to this property of the CNS, showing the most recent works in this field of research.

\subsection{Cell Signaling Molecules}

\section{Plasticity molecules}

To analyze the role of neural cell adhesion molecule (NCAM) in brain plasticity, Aonurm-Helm and colleagues (2008) used a NCAM-deficient (NCAM-/-) mice exhibiting depression-like behavior and reduced adult neurogenesis in the dentate gyrus, with reduced levels of the phosphorylated cAMP response element-binding protein (pCREB) in the hippocampus. They used a treatment with a peptide termed fibroblast growth loop (FGL), derived from the NCAM binding site for the fibroblast growth factor (FGF) receptor, what was able to reverse the depression-like signs in NCAM-/- mice. The FGL administered acutely or repeatedly reduced depression-like behavior in NCAM- -- mice, enhanced survival of the newly born neurons in NCAM- -- mice and increased the levels of pCREB in both $\mathrm{NCAM}+/+$ and $\mathrm{NCAM}-/-$ mice. In conclusion, this study has demonstrated that NCAM deficiency in mice results in a depression-like phenotype which can be reversed by the acute or repeated administration of FGL. The results also suggest a role of the deficit in NCAM signaling through the FGF receptor in depression [11].

\section{Transcriptions factors}

A review study showed a set of transcription factors, secreted molecules and plasma membrane markers that are differentially regulated during differentiation [7]. Pathway analysis in this work highlights alterations in insulin growth factor (IGF), Wnt and transforming growth factor $\mathrm{b}$ (TGF-B) signaling cascades.

In the established adult rat neural stream cell (NSC) culture, fibroblast growth factor-2 (FGF-2) promotes selfrenewal by increasing proliferation and inhibiting spontaneous differentiation of adult NSCs, accompanied with activation of MAPK (Mitogen-activated protein kinase) and PLC (Phospholipase C) pathways. Using a molecular genetic approach, Ma and collaborators (2009) demonstrated that activation of FGF receptor 1 (FGFR1), largely through two key cytoplasmic amino acid residues that are linked to MAPK and PLC activation, suffices to promote adult NSC self-renewal. They showed that canonical MAPK, Erk1/2 (extracellular signal-regulated kinases) 
activation, is both required and sufficient for the NSC expansion and anti-differentiation effects of FGF-2. In contrast, PLC activation is integral to the maintenance of adult NSC characteristics, including the full capacity for neuronal and oligodendroglial differentiation. This study revealed two amino acid residues in FGFR1 with linked downstream intracellular signal transduction pathways that are essential for maintaining adult NSC self-renewal [12].

Transcription factors are crucial in orchestrating the correct cell-specific and temporal expression of all factors involved in these signaling networks. Aberrant expression of these factors can lead to abnormal brain development if this occurs during embryogenesis [8]. The transcription factor CREB has been implicated in signaling pathways relevant for pathogenesis and therapy of depression. CREB is up regulated and activated in the hippocampus by chronic antidepressant treatment, similarly as neurogenesis [13].

Another work, using an ablated CREB-pathway neurogenic lineage, showed that loss of CREB signaling resulted in increased cell death and loss of expression of the neurogenic transcription factor Pax 6, and of a subset of neuronal proteins in migrating neurons of the RMS [14]. Moreover, post-migratory neurons in the OB displayed impaired dendritic development. These results demonstrate an essential role for CREB signaling in maturation of newborn neurons in the $\mathrm{OB}$ and uncover a novel role for CREB signaling in the survival and maintenance of neuronal gene expression during the early stages of SVZ/OB.

Fiorentini and others (2010) used a double transgenic (Tg) CRND8 mice (over expressing the Swedish and Indiana mutations in the human amyloid precursor protein) that shows a decreased neurogenesis in the SGZ of $\mathrm{Tg}$ mice compared to non- $\mathrm{Tg}$ mice. The decrease of hippocampal neurogenesis was accompanied by behavioral deficits and worsened with age and pathology severity. The differentiation into neurons and maturation of the proliferating cells were also markedly impaired in the $\mathrm{Tg}$ mice. Lithium treatment to Tg mice significantly stimulated the proliferation and neuron fate specification of newborn cells and fully counteracted the transgene-induced impairments of cognitive functions. The drug, by the inhibition of GSK-3b (Glycogen synthase kinase-3b) and subsequent activation of $\mathrm{Wnt} / \beta$-catenin signaling promoted hippocampal neurogenesis. Finally, the data showed that the lithium's ability to stimulate neurogenesis and cognitive functions was lost in the aged Tg mice, thus indicating that the lithium-induced facilitation of neurogenesis and cognitive functions declines as brain $\mathrm{Ab}$ deposition and pathology increases [9].

Activity requires soluble amyloid precursor protein (sAPP) to enhance neurite outgrowth of young neurons differentiating from neural stem cells. Inhibition of sAPP secretion and anti-APP antibodies both abolished the effect of depolarization on neurite outgrowth, whereas exogenous SAPPa, similar to depolarization, induced neurite elongation. Depolarization and $\mathrm{SAPPa}$ both required active N-methyl-d-aspartic acid receptor (NMDAR) and mitogen-activated protein kinase (MAPK)/extracellular signal-regulated kinase (ERK) recruitment to induce neurite outgrowth. However, depolarization and SAPPa played different roles in modulating this signaling cascade. Depolarization induced ERK phosphorylation with fast kinetics via activation of NMDAR. By contrast, acute application of sAPPa did not lead to ERK activation. However, continuous generation of SAPPa was necessary for depolarization-induced ERK phosphorylation, indicating that SAPPa promotes MAPK/ERK recruitment by an indirect mechanism. In addition, they found that blockade of NMDAR down-regulated APP expression, whereas depolarization increased SAPPa, suggesting that activity may also act upstream of sAPP signaling by regulating the amount of cellular APP and extracellular sAPPa. Finally, they showed that soluble amyloid precursor-like protein 2 (sAPLP2), but not sAPLP1, is functionally redundant to SAPP in promoting neurite outgrowth and that soluble members of the APP family require membranebound APP to enhance neurite outgrowth [15].

Cultured adult neural stem cells express a considerable variety of nucleotide receptors and that nucleotides and epidermal growth factor (EGF) induce converging intracellular signaling pathways that carry potential for synergism in the control of neural stem cell proliferation and cell survival. They investigate the role of EGF and the nucleotides ATP (Adenosine triphosphate), ADPbS (adenosine 5-( $\beta$-thio) diphosphate) and UTP (Nucleoside triphosphate) in neural stem cell migration. Nucleotides and EGF induce the formation of stress fibres, an increase in the cortical actin cytoskeleton and in cell spreading. This is associated with increased phosphorylation of Akt (Protein Kinase B - PKB) and focal adhesion kinase. Nucleotides and EGF acting as paracrine or autocrine signaling substances can be of relevance for structuring and maintaining the cytoarchitecture of the SVZ and the stream of neuroblasts migrating to the OB [10].

The insulin-like growth factor receptor type 1 (IGF1R) signaling pathway is activated in the mammalian nervous system from early developmental stages. Its major effect on developing neural cells is to promote their growth and survival. This pathway can integrate its action with signaling pathways of growth and morphogenetic factors that induce cell fate specification and selective expansion of specified neural cell subsets. This suggests that during developmental and adult neurogenesis cellular responses to many signaling factors, including ligands of Notch, 
sonic hedgehog, fibroblast growth factor family members, ligands of the epidermal growth factor receptor, bone morphogenetic proteins and Wingless and Int-1, may be modified by co-activation of the IGF1R. Modulation of cell migration is another possible role that IGF1R activation may play in neurogenesis [16].

Bateman and McNeill (2006) demonstrated that IR/ IGF1r null animals show defects in CNS development. The mechanism of action by which insulin/IGF-stimulated differentiation may occur through activation of the Ras/MAPK pathway. The contribution of insulin/IGF signaling to neurogenesis may be context and/or cell type specific; however, the importance of fine spatiotemporal control of neuronal differentiation means that understanding the role of this pathway is of major importance. Small alterations in the wiring of the brain can have profound consequences on function, and there are abundant data to suggest that the cues for axonal guidance alter over developmental time. To generate a structure of such intricacy as the brain, growth and differentiation must be coordinated, and the insulin/IGF signaling pathway appears to have just such a function [17]. Insulin/IGF signaling has a conserved role in both proliferation and neuronal differentiation.

\subsection{Wnt- $\beta$-Catenin or Canonical and Non-Canonical Wnt Signaling Pathway}

Wnt signaling is involved in the development of the brain and spinal cord, therefore little is known about the roles of Wnt in the adult nervous system and implicated in the effects of exercise, sensory deprivation and ageing on the CNS $[1,18]$.

\subsection{GABA ( $y$-Aminobutyric Acid)}

The GABA-induced excitation via GABAA receptor activation mediated depolarization regulates synaptic development of young neurons. Thus in newly generated granule neurons in the adult dentate gyrus, providing evidence that GABA drives synaptic integration of newborn neurons in adults. Whereas, there are diverse roles of GABA, seem to depend on cell-intrinsic properties and on extrinsic factors, from proliferation, migration, synaptogenesis and circuit formation [19].

\subsection{Neurotrophin}

Neurotrophins (NTs) are a family of trophic factors critical for the survival and development of neurons within the peripheral and central nervous systems. Members of the family include the prototypic member nerve growth factor (NGF), brain-derived neurotrophic factor (BDNF), NT-3 and NT-4/5 in mammals, and NT-6/7 in fish species. The actions of NTs are mediated by tropomyosinre- ceptor-kinase (Trks), a family of receptor tyrosine kinases and $\mathrm{p} 75$, the low affinity NT receptor. Binding of NTs to Trk receptors such as TrkA, TrkB, or TrkC induces rapid tyrosine phosphorylation of the receptors, resulting in their trans-activation and thereby activating multiple intracellular signal transduction pathways including Ras/ MAPK (RAt Sarcoma/mitogen-activated protein kinase), phosphatidylinositol 3-kinase (PI3K) and phospholipase C. Strikingly, some examples of genes regulated by either NGF or cAMP (Cyclic adenosine monophosphate) including ephexin 1, G-protein-coupled receptor 50, prepronociceptin, voltage-gated sodium channel type 6polypeptide, corticotrophin-releasing hormone, secretin receptor, tachykinin 1 , annexin $\mathrm{A} 1$, and bradykinin receptor B2 showed comparable levels of regulation, suggesting that these NGF-induced genes could be induced through a cAMP-dependent pathway [20].

\subsection{Endocannabinoid Pathway}

The endocannabinoid system is a neuromodulatory system in the brain and pharmacological enhancement of neurotransmission involving endocannabinoid has been shown to inhibit stress-induced activation of the hypothalamus-pituitary-adrenal (HPA) axis. Moreover, inhibition of endocannabinoid reuptake exhibits antianxiety and antidepressant properties and suggest that this system may act as a buffer in the stress response. Recent evidence, both in vitro and in vivo studies, have indicated that activation of CB1 (cannabinoid type 1) receptors facilitates cell proliferation and neurogenesis in the hippocampus, furthermore, can suppress the neuroendocrine response to stress [21].

Recently shown that chronic $\Delta 9$-Tetrahydrocannabinol (THC) administration in females, adolescent rats induces subtle but lasting alterations in the emotional circuit ending in depressive-like behavior at adulthood, for example, increased immobility in the forced swim test, decreased sucrose preference, suggestive of anhedonia, and a deficit in spatial working memory. Note also at the level cellular, increased and decreased activation in the nucleus accumbens and hippocampus, respectively. Thus suggests the hypothesis that reduction in $\mathrm{CB} 1$ receptor signaling in the amygdala, nucleus accumbens and in the ventral tegmental area reduced activity of the endocannabinoid system could predispose to depression [22].

The endocannabinoid system participates in the regulation of physical activity, although its role is not yet fully understood. A recent work studied the impact of endocannabinoid signaling on voluntary wheel running in mice and discussed potential mechanisms involved such as hippocampal neurogenesis. Endocannabinoids might be involved most likely via CB1 receptors [23]. 


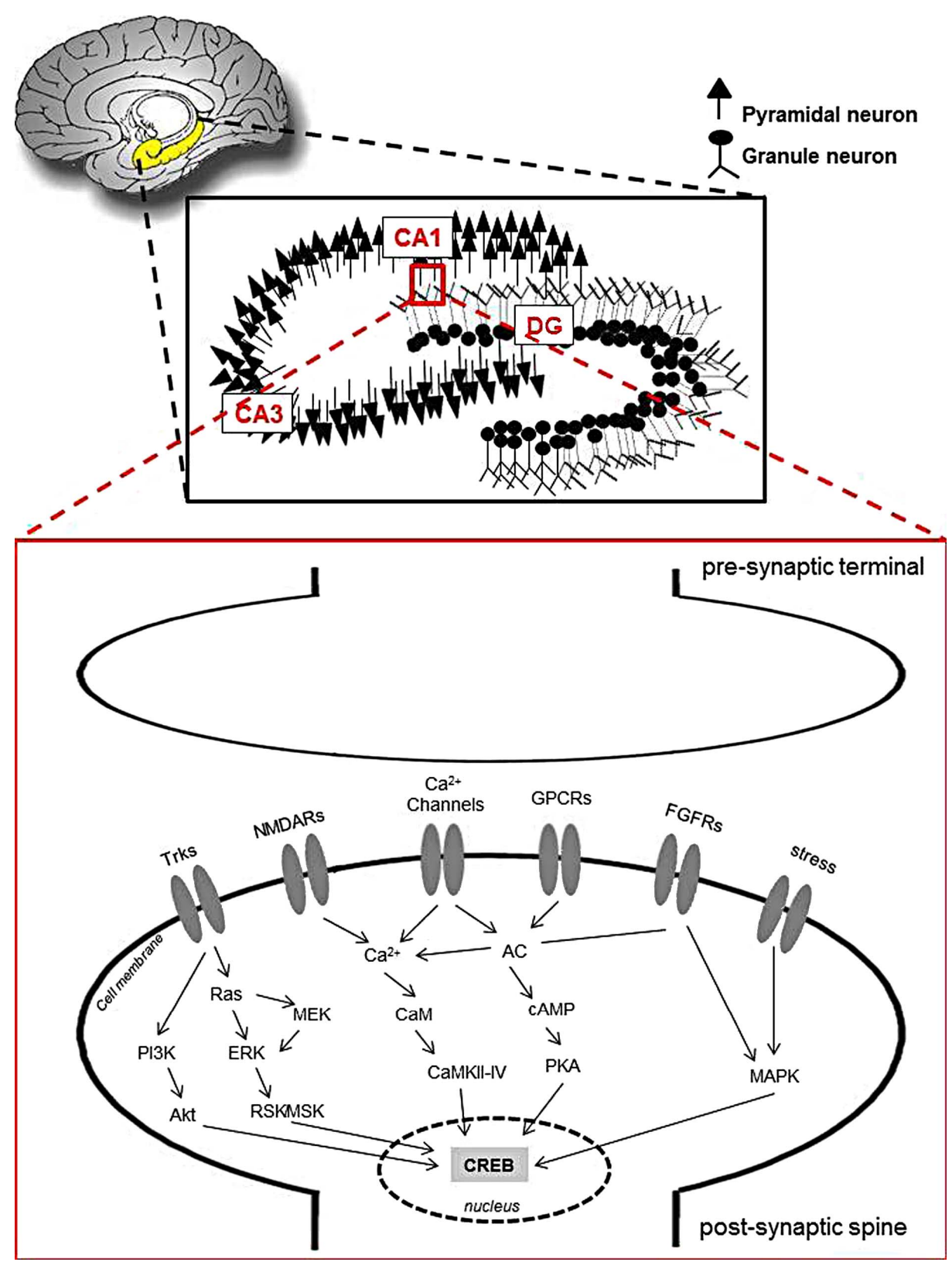

Figure 1. Outline of the "macro" to "micro". Above and below: the location of the hippocampus with more detailed regions CA1, CA3 and dentate gyrus (DG). Below: synapse and signaling pathways of neurogenesis.

\section{Macro: Stress and Depression}

A number of environmental stimuli, such as social stress, lead to a downregulation of neurogenesis and cognitive functions [24]. Excess of glucocorticoids triggers some pathological events that may be reduce dendritic arborization in hippocampal neurons and can also cause hippocampal dentate gyrus neuronal death. This link between hypothalamic-pituitary-adrenal (HPA) axis and hippocampal atrophy may shed new lights into the pathophysiology of mood disorders and stress-related cognitive dysfunctions. Furthermore, the canonical Wnt pathway may be effective in preventing hippocampal damage as- sociated with stress related disorders, including major depression [25].

Furthermore, the psychosocial stress reduces the proliferation of hippocampal NSCs and the survival of newly born neurons in the hippocampus. Likewise, conditions that suppress hippocampal neurogenesis, such as, have been linked to amplified depressive-like behavior and/or cognitive dysfunction as result of unpredictable chronic stress (UCS) or increased levels of stress hormones. However, stress and glucocorticoids are among the most potent inhibitors of neurogenesis in the adult dentate gyrus [6]. 
There are two types of corticoid receptors, type I or mineralocorticoids receptors (MR) and type II or glucocorticoids receptor (GR). GR has been found as a common mechanism for stress dependent changes in brain function and a potential target of antidepressant drugs. The glucocorticoids signaling appear to be involved both in up- and down-neurogenesis in the hippocampus [26]. The excessive glucocorticoid signaling participates of the reduction of neurons in the hippocampus, while the antidepressant treatment is also involved with this signaling. Considering the critical role of the GR in HPA axis hyperactivity and in mediating the effects of glucocorticoids on brain plasticity and mood, it is not surprising that the GR has been found to be a common mechanism for stress dependent changes in brain function and a potential target of antidepressant drugs [26].

However, in some animal models of mild or moderate stress was noted the opposite effects of severe stress. Thus, there was an increase in neurogenesis, as well as decreased anxiety and depressive behavior, and an improvement in spatial memory retention, absence of the neuroinflammation and neurodegeneration [27].

There is considerable evidence for a cyclic AMP (cAMP)/protein kinase A (PKA) dependent mechanism of GR regulation. PKA induces GR-transactivation in cells that lack endogenous cAMP response element binding protein (CREB) [26].

The neurogenesis hypothesis of depression postulates that a decrease in the production of newborn granule cells in the dentate gyrus of the hippocampus is related to the pathophysiology of depression and that enhanced hippocampal neurogenesis is required for the behavioral effect of antidepressant treatments [5].

Antidepressants not only alleviate depressive symptoms and normalize HPA axis hyperactivity, they also protect from neuronal cell death and from reduction in adult hippocampal neurogenesis. These neuroprotective effects have been suggested to be mediated, at least in part, by elevated BDNF levels upon antidepressant treatment [26]. The antidepressants increase human hippocampal NPCs and angiogenesis selectively in the DG [28].

In the last two decades, several studies demonstrated that the stimulation of adult cell proliferation and neurogenesis in the DG is regulated by genetic and epigenetic factors. Indeed, in the hippocampus, BDNF has been extensively shown to be increased in response to exercise. In adult neurogenesis the Ras-mediated extracellular signalregulated cascade (ERK) pathway and endogenously regulated Ras is coexpressed with permanently activated Ras in neurons is considered as a major BDNF/TrkB intracellular signaling pathway in neurons leading to enhanced morphological differentiation and survival of mature neurons [24].

\section{Closing Remarks and Future Directions}

It is known that activity modulates neuronal differentiation in the adult brain but the signaling mechanisms underlying this process remain to be identified. The findings in this work provide novel insights into the molecular mechanism regulating adult neural stem cells self renewal, and pose implications for using this information in potential therapeutic applications.

In this work, the role of CREB in this signaling was highlighted. CREB (cAMP response element-binding) is a cellular transcription factor and it binds to certain DNA sequences called cAMP response elements (CRE), namely positive and negative regulators of gene transcription which include neurotrophin, BDNF, tyrosine hydroxylase, and many neuropeptides (such as somatostatin, enkephalin, VGF, and corticotropin-releasing hormone). Increases in the concentration of either calcium or cAMP can trigger the phosphorylation and activation of CREB. This transcription factor is a component of intracellular signaling events that regulate a wide range of biological functions in neuronal plasticity, long-term memory formation in the brain, spermatogenesis to circadian rhythms. At the end of the post-synaptic stimuli such as activation of receptors Trks, NMDAs and FGF, as well as calcium channels and stress, all culminate in ways that lead to the nucleus and thus phosphorylate the transcription factor, CREB, as can be seen in Figure 1.

Overall, the studies of adult neurogenesis are still in their infancy, because most of them explore only one stage of this process. Importantly, given the built-in homeostatic mechanisms that act at each stage during the progression from stem cells to mature neurons (proliferation, differentiation, maturation, survival), it is very difficult to extrapolate the efficiency of a drug on adult neurogenesis from analysis of one stage alone. Here, we review the most significant data on adult neurogenesis, and it is important to study each stage of this process [5].

Further characterization of the molecular components could provide greater insight into the mechanisms involved in the regulation of neurogenesis in the adult brain. The challenge for the future is to understand molecularly how proliferation and differentiation are coordinated by a single pathway [17].

\section{Acknowledgements}

This work was supported by grants from CNPq, CAPES and FAPERJ. The authors thank Luciana Nogaroli for useful comments.

\section{REFERENCES}

[1] N. C. Inestrosa and E. Arenas, "Emerging Roles of Wnts in the Adult Nervous System," Nature Reviews Neuro- 
science, Vol. 11, No. 2, 2010, pp. 77-86. http://dx.doi.org/10.1038/nrn2755

[2] G. L. Ming and H. Song, "Adult Neurogenesis in the Mammalian Central Nervous System," Annu Rev Neurosci, Vol. 28, 2005, pp. 223-250. http://dx.doi.org/10.1146/annurev.neuro.28.051804.10145 $\underline{9}$

[3] H. C. Yan, et al., "Fuzi Polysaccharide-1 Produces Antidepressant-Like Effects in Mice," The International Journal of Neuropsychopharmacology/Official Scientific Journal of the Collegium Internationale Neuropsychopharmacologicum, Vol. 13, No. 5, 2010, pp. 623-633.

[4] H. C. Yan, et al., "Fuzi Polysaccharide-1 Produces Antidepressant-Like Effects in Mice," International Journal of Neuropsychopharmacology, Vol. 13, No. 5, 2010, pp. 623-633. http://dx.doi.org/10.1017/S1461145709990733

[5] D. J. David, et al., "Implications of the Functional Integration of Adult-Born Hippocampal Neurons in AnxietyDepression Disorders," Neuroscientist, Vol. 16, No. 5, 2010, pp. 578-591. http://dx.doi.org/10.1177/1073858409360281

[6] B. Czeh and P. J. Lucassen, "What Causes the Hippocampal Volume Decrease in Depression? Are Neurogenesis, Glial Changes and Apoptosis Implicated?" European Archives of Psychiatry and Clinical Neurosciences, Vol. 257, No. 5, 2007, pp. 250-260.

http://dx.doi.org/10.1007/s00406-007-0728-0

[7] T. P. Bonnert, et al., "Molecular Characterization of Adult Mouse Subventricular Zone Progenitor Cells during the Onset of Differentiation," European Journal of Neuroscience, Vol. 24, No. 3, 2006, pp. 661-675. http://dx.doi.org/10.1111/j.1460-9568.2006.04912.x

[8] S. Dworkin and T. Mantamadiotis, "Targeting CREB Signalling in Neurogenesis," Expert Opinion on Therapeutic Targets, Vol. 14, No. 8, 2010, pp. 869-879. http://dx.doi.org/10.1517/14728222.2010.501332

[9] A. Fiorentini, et al., "Lithium Improves Hippocampal Neurogenesis, Neuropathology and Cognitive Functions in APP Mutant Mice," PLoS One, Vol. 5, No. 12, 2010, p. e14382. http://dx.doi.org/10.1371/journal.pone.0014382

[10] I. Grimm, S. N. Ullsperger and H. Zimmermann, "Nucleotides and Epidermal Growth Factor Induce Parallel Cytoskeletal Rearrangements and Migration in Cultured Adult Murine Neural Stem Cells," Acta Physiologica (Oxford), Vol. 199, No. 2, 2010, pp. 181-189.

http://dx.doi.org/10.1111/j.1748-1716.2010.02092.x

[11] A. Aonurm-Helm, et al., "Depression-Like Behaviour in Neural Cell Adhesion Molecule (NCAM)-Deficient Mice and Its Reversal by an NCAM-Derived Peptide," FGL European Journal of Neuroscience, Vol. 28, No. 8, 2008, pp. 1618-1628. http://dx.doi.org/10.1111/j.1460-9568.2008.06471.x

[12] D. K. Ma, et al., "Molecular Genetic Analysis of FGFR1 Signalling Reveals Distinct Roles of MAPK and PLC Gamma1 Activation for Self-Renewal of Adult Neural Stem Cells," Molecular Brain, Vol. 2, 2009, p. 16. http://dx.doi.org/10.1186/1756-6606-2-16

[13] P. Gass and M. A. Riva, "CREB, Neurogenesis and Depression,” Bioessays, Vol. 29, No. 10, 2007, pp. 957-961. http://dx.doi.org/10.1002/bies.20658

[14] S. Herold, et al., "CREB Signalling Regulates Early Survival, Neuronal Gene Expression and Morphological Development in Adult Subventricular Zone Neurogenesis," Molecular and Cellular Neuroscience, Vol. 46, No. 1, 2011, pp. 79-88.

http://dx.doi.org/10.1016/j.mcn.2010.08.008

[15] N. Gakhar-Koppole, et al., "Activity Requires Soluble Amyloid Precursor Protein Alpha to Promote Neurite Outgrowth in Neural Stem Cell-Derived Neurons via Activation of the MAPK Pathway," European Journal of Neuroscience, Vol. 28, No. 5, 2008, pp. 871-882. http://dx.doi.org/10.1111/j.1460-9568.2008.06398.x

[16] A. Annenkov, "The Insulin-Like Growth Factor (IGF) Receptor Type 1 (IGF1R) as an Essential Component of the Signalling Network Regulating Neurogenesis," Molecular Neurobiology, Vol. 40, No. 3, 2009, pp. 195-215. http://dx.doi.org/10.1007/s12035-009-8081-0

[17] J. M. Bateman and H. McNeill, "Insulin/IGF Signalling in Neurogenesis," Cellular and Molecular Life Sciences, Vol. 63, No. 15, 2006, pp. 1701-1705. http://dx.doi.org/10.1007/s00018-006-6036-4

[18] C. Matute and F. Cavaliere, "Neuroglial Interactions Mediated by Purinergic Signalling in the Pathophysiology of CNS Disorders," Seminars in Cell \& Developmental Biology, Vol. 22, No. 2, 2011, pp. 252-259.

http://dx.doi.org/10.1016/j.semcdb.2011.02.011

[19] D. D. Wang and A. R. Kriegstein, "Defining the Role of GABA in Cortical Development," Journal of Physiology, Vol. 587, No. 9, 2009, pp. 1873-1879.

[20] Y. P. Ng, et al., "Differential and Synergistic Effect of Nerve Growth Factor and cAMP on the Regulation of Early Response Genes during Neuronal Differentiation," Neurosignals, Vol. 17, No. 2, 2009, pp. 111-120.

http://dx.doi.org/10.1159/000197391

[21] M. N. Hill, et al., "Endocannabinoids Modulate StressInduced Suppression of Hippocampal Cell Proliferation and Activation of Defensive Behaviours," The European Journal of Neuroscience, Vol. 24, No. 7, 2006, pp. 18451849. http://dx.doi.org/10.1111/j.1460-9568.2006.05061.x

[22] N. Realini, et al., "Chronic URB597 Treatment at Adulthood Reverted Most Depressive-Like Symptoms Induced by Adolescent Exposure to THC in Female Rats," Neuropharmacology, Vol. 60, No. 2-3, 2011, pp. 235-243. http://dx.doi.org/10.1016/j.neuropharm.2010.09.003

[23] J. Fuss and P. Gass, "Endocannabinoids and Voluntary Activity in Mice: Runner's High and Long-Term Consequences in Emotional Behaviors," Experimental Neurology, Vol. 224, No. 1, 2010, pp. 103-105. http://dx.doi.org/10.1016/j.expneurol.2010.03.016

[24] P. Lafenetre, et al., "Exercise Can Rescue Recognition Memory Impairment in a Model with Reduced Adult Hippocampal Neurogenesis," Frontiers in Behavioral Neuroscience, Vol. 3, 2010, p. 34.

[25] F. Matrisciano, et al., "Induction of the Wnt Antagonist Dickkopf-1 Is Involved in Stress-Induced Hippocampal Damage," PLoS One, Vol. 6, No. 1, 2011, p. e16447. http://dx.doi.org/10.1371/journal.pone.0016447 
[26] C. Anacker and C. M. Pariante, "Can Adult Neurogenesis Buffer Stress Responses and Depressive Behaviour?" Molecular Psychiatry, 2011.

[27] V. K. Parihar, et al., "Predictable Chronic Mild Stress Improves Mood, Hippocampal Neurogenesis and Memory," Molecular Psychiatry, Vol. 16, No. 2, 2011, pp. 171-183. http://dx.doi.org/10.1038/mp.2009.130

[28] M. Boldrini, et al., "Hippocampal Angiogenesis and Progenitor Cell Proliferation Are Increased with Antidepressant Use in Major Depression," Biological Psychiatry, Vol. 72, No. 7, 2012, pp. 562-571.

http://dx.doi.org/10.1016/j.biopsych.2012.04.024 